Journal of Animal and Veterinary Advances 9 (10): 1463-1468, 2010

ISSN: $1680-5593$

(C) Medwell Journals, 2010

\title{
Sequence Variation in Toxoplasma gondii MIC4 Gene and Protective Effect of an MIC4 DNA Vaccine in a Murine Model Against Toxoplasmosis
}

\author{
${ }^{1}$ G.H. Peng, ${ }^{1}$ Z.G. Yuan, ${ }^{1}$ D.H. Zhou, ${ }^{1}$ X.H. He, ${ }^{1}$ C. Yan, ${ }^{1}$ C.C. Yin, \\ ${ }^{1}$ Y. He, ${ }^{1}$ R.Q. Lin, ${ }^{1,2}$ H.Q. Song and ${ }^{1-3}$ X.Q. Zhu \\ ${ }^{1}$ College of Veterinary Medicine, South China Agricultural University, 483 Wushan Street, \\ Tianhe District, Guangzhou, Guangdong Province 510642, China \\ ${ }^{2}$ State Key Laboratory of Veterinary Etiological, Biology, Parasitology of Gansu Province, \\ Lanzhou Veterinary Research Institute, CAAS, Lanzhou, Gansu Province 730046, China \\ ${ }^{3}$ College of Animal Science and Technology, Yunnan Agricultural University, \\ Kunming, Yunnan Province 650201, China
}

\begin{abstract}
Toxoplasma gondii infects nearly one third of the total population of the world, as well as warm blooded animals causing serious public health problems and economic losses in the world. Micronemes plays a key role in the invasion process of $T$. gondii which are used for host cell recognition, binding and motility. In this research, the researchers examined sequence variation in the microneme protein 4 (MIC4) gene sequences of $12 \mathrm{~T}$. gondii isolates and reference strains from different hosts and geographical locations, then constructed the DNA vaccine expressing MIC 4 of $T$. gondii evaluated its immune response induced in Kunming mice. The results demonstrated that sequence variation in MIC 4 among different $T$. gondii isolates was low, which is a useful feature as a vaccine candidate. Immunization of mice with pVAX-MIC4 induced strong immune responses in mice as shown by significant lymphocyte proliferation, cytokine production and antibody responses as well as increased survival time of the immunized mice after challenge with tachyzoites of the virulent $T$. gondii $\mathrm{RH}$ strain, demonstrating that $T$. gondii $\mathrm{MIC} 4$ is a potential vaccine candidate against toxoplasmosis.
\end{abstract}

Key words: Toxoplasma gondii, toxoplasmosis, Microneme protein 4 (MIC4), immunity, DNA vaccine, mice, variation

\section{INTRODUCTION}

Toxoplasma gondii is an obligate intracellular protozoan capable of infecting humans and a variety of mammals and birds (Montoya and Liesenfeld, 2004; Weiss and Dubey, 2009) with a worldwide distribution. T. gondii is considered the most successful parasite because it infects one-third of human world population, causing considerable diseases in immuno-compromised patients and pregnant women (Montoya and Liesenfeld, 2004). Moreover, toxoplasmosis can result in serious economic losses and causes abortions, stillbirths and neonatal deaths in all kinds of livestock (Buxton, 1998).

Despite the current knowledge of immunology, pathology and genetics related to the parasite, the effective treatment and a safe vaccine for prevention of the infection in both humans and animals are still not available (Zhang et al., 2007). Thus, the development of an effective vaccine against $T$. gondii would be extremely valuable for the effective control of $T$. gondii infection in humans and animals. Micronemal proteins (MICs) are thought to be critical ligands determining host cell specificity at the time of invasion.

$\mathrm{MIC}$ adhesive proteins contribute to binding host cell receptors and blocking micronemal secretion dramatically reduces invasion (Carruthers et al., 1999). These proteins, which often cluster into multiunit complexes are actively discharged from parasites upon the apical attachment to a host cell and play a role in host cell attachment and penetration (Tomley and Soldati, 2001).

Several studies of DNA-based vaccines against toxoplasmosis have been conducted, mainly with mice and various $T$. gondii antigens such as membrane associated surface antigen SAG1 (Angus et al., 2000; Nielsen et al., 1999), rhoptry proteins ROP1 (Chen et al., 2001) and ROP2 (Leyva et al., 2001; Vercammen et al., 2000), excreted-secreted dense-granule proteins GRAl

Corresponding Author: X.Q. Zhu, College of Veterinary Medicine, South China Agricultural University, 483 Wushan Street, Tianhe District, Guangzhou, Guangdong Province 510642, The people's Republic of China 
(Scorza et al., 2003; Vercammen et al., 2000), GRA4 (Desolme et al., 2000) and GRA7 (Vercammen et al., 2000), Micronemal proteins MIC1, MIC3, MIC4 and MIC6 (Lourenco et al., 2006; Peng et al., 2009; Xiang et al., 2009; Wang et al., 2009). All of them showed the protective effect to some extent. The objectives of the present study were to examine sequence variation in the MIC 4 gene and evaluate the protective effect of a MIC4 DNA vaccine (expressed in the eukaryotic expression vector $\mathrm{pVAX} I$ ) in a murine model against $T$. gondii.

\section{MATERIALS AND METHODS}

Mice, parasites, isolation of genomic DNA and preparation of Soluble Tachyzoite Antigens (STAg): Female Kunming mice used in this experiment were purchased from Southern Medical University Laboratory Animal Center in China. All experimental procedures were conducted according to institutional guidelines for animal ethics. All mice were maintained under specific-pathogen free conditions and were at 5-6 weeks of age when immunizations were initiated. Tachyzoites of $T$. gondii ( $\mathrm{RH}$ strain) were conserved well in liquid nitrogen in the laboratory. The researchers dissolved them in $38^{\circ} \mathrm{C}$ waterbath and infected Kunming mice intraperitoneally and harvested tachyzoites from the peritoneal fluid of mice after $72 \mathrm{~h}$. After they were washed by centrifugation and then suspended in sterile Phosphate-Buffered Saline (PBS), the tachyzoites of $T$. gondii were used for challenge of immunized mice. Genomic DNA (gDNA) was extracted from the tachyzoites stored at $-80^{\circ} \mathrm{C}$ by sodium dodecyl-sulphate/proteinase $\mathrm{K}$ treatment (Zhu et al., 2002), column-purified using Wizard ${ }^{\mathrm{TM}}$ DNA Clean-Up System (Promega) and eluted into $50 \mu \mathrm{L} \mathrm{H}_{2} \mathrm{O}$ according to the manufacturer's recommendations. DNA samples were stored at $-20^{\circ} \mathrm{C}$ until use.

For the preparation of STAg, the tachyzoites were collected from the peritoneal fluids, washed by centrifugation and then suspended in sterile Phosphate Buffered Saline (PBS) and sonicated. The sonicate was centrifuged at $2100 \times \mathrm{g}$ for $15 \mathrm{~min}$ and the supernatant containing STAg was kept at $-80^{\circ} \mathrm{C}$ until further use.

Enzymatic amplification, sequencing and analysis: The MIC4 gene was amplified with a pair of primers (MIC4, forward primer: 5'-GAATTCATGAGAGCGTCGCTC-3' and reverse primer: 5'-CTCGAGTCATTCTGTGTCTTTCGC-3'. PCR reactions $(25 \mu \mathrm{L})$ were performed in $2 \mathrm{mM}$ of $\mathrm{MgCl}_{2}$, $2.5 \mu \mathrm{M}$ of each primer, $2.5 \mu \mathrm{L}$ Ex Taq buffer, $0.2 \mathrm{mM}$ of each dNTPs, $1.25 \mathrm{U}$ of Ex Taq DNA polymerase (TAKARA), $2 \mu \mathrm{L}$ of DNA sample in a thermocycler (Biometra) under the following conditions: initial denaturation at $94^{\circ} \mathrm{C}$ for $4 \mathrm{~min}$ followed by 36 cycles of $94^{\circ} \mathrm{C}, 1 \mathrm{~min}$ (denaturation), $62^{\circ} \mathrm{C}, 1 \mathrm{~min}$ (annealing), $72^{\circ} \mathrm{C}$, $2 \mathrm{~min}$ (extension) and a final extension of $72^{\circ} \mathrm{C}$ for $7 \mathrm{~min}$. Samples with host DNA were included in each amplification run as host controls. Samples without genomic DNA were included in each amplification run as negative controls. Each amplicon $(5 \mu \mathrm{L})$ was examined by agarose gel electrophoresis to validate amplification efficiency. The DL2000 marker (TAKARA) was used to estimate the size of $\mathrm{MIC} 4$ amplicons.

PCR products were purified using spin columns (Wizard ${ }^{\text {Ts }}$ PCR-Preps DNA Purification System, Promega) and the purified PCR products were ligated with pGEM-T Easy plasmid vector (Promega) according to manufacturer's instructions. The recombinant plasmid was transformed into JM109 competent cells (Promega), positive transformants containing recombinant plasmids were selected and the plasmid DNA extracted using Wizard $^{\mathrm{TM}}$ Plus Minipreps DNA Purification System (Promega). Cell cultures with confirmed recombinant plasmid were sequenced using an ABI 377 automated DNA sequencer (BigDye Terminator Chemistry).

Sequences of the MIC4 gene were aligned using the computer program ClustalX 1.81 (Thompson et al., 1997), genetic distance calculation was performed using PUZZLE 4.1 (Strimmer and Von Haeseler, 1996), sequence homology analysis was performed and the nucleotide composition, transition and transversion were accounted using the Megalign program in the software DNA Star version 5.0 .

Construction of the eukaryotic expression plasmid: After generation of pGEM-MIC6 as earlier, the fragment of MIC6 was inserted into the eukaryotic expression vector pVAX I by double digestion with restriction enzymes EcoR I/Xho I and ligation was finally done using $\mathrm{T}_{4}$ DNA ligase (Promega). The plasmid that gained was named pVAX-MIC4. The concentration of pVAX-MIC4 was determined by spectrophotometer at $\mathrm{OD}_{260}$ and $\mathrm{OD}_{280}$.

Expression of MIC4 in vitro: Marc-145 cells were transfected with a kind of eukaryotic expression plasmid or an empty vector (control plasmid) using lipofectamine ${ }^{\mathrm{TM}} 2000$ reagent (Invitrogen) according to the manufacturer's recommendations. After $48 \mathrm{~h}$, the cells that transfected were fixed with cool acetone for $30 \mathrm{~min}$ and MIC4 expression was detected using the indirect Immunofluorescence Assay (IFA) with anti- $T$. gondii polyclonal antiserum (Goat) and a FITC-labeled donkey-anti-goat IgG (Proteintech Group Inc, Chicago, USA). Evans blue (Fisher) was included in the secondary antibody solution as a counterstain. Coverslips were 
rinsed three times with $\mathrm{PBS}$. The monolayers binding the marker were covered with glycerine and examined for specific fluorescence under a Zeiss Axioplan fluorescence microscope.

DNA imm unization: Eighty Kunming mice were randomly divided into four groups of 20 mice per group. Mice in Group I was injected intramuscularly with PBS as control, group II with empty pVAX I vector also as control, group III with $100 \mu \mathrm{g}$ of pVAX-MIC4 DNA suspended in $100 \mu \mathrm{L}$ sterile PBS. Group IV served as non-treatment control. Mice were immunized using the same protocol on weeks 0,2 and 4. The blood of mice in each group was collected by retro-orbital plexus puncture on days 13, 27 and sera were stored at $-20^{\circ} \mathrm{C}$ until use.

IgG determination: Serum samples from immunized mice were tested using anti- $T$. gondii IgG ELISA Kit according to the manufacture's recommendations (Combined Biotech CO., LTD, Shenzhen, China). Plates were coated overnight at $4^{\circ} \mathrm{C}$ with $1 \mu \mathrm{g} \mathrm{mL} \mathrm{m}^{-1}$ solution of STAg in carbonate buffer $\mathrm{pH}$ 9.2. After washing three times (each time for $3 \mathrm{~min}$ ) with PBS containing 0.05\% Tween-20 (PBST-20), sera diluted in 1\% BSA-PBST-20 (1:100) were applied to the wells and incubated for $30 \mathrm{~min}$ at $37^{\circ} \mathrm{C}$. After washing, bound antibodies were incubated with $50 \mu \mathrm{L}$ horseradish peroxidase-conjugated goat anti-mouse $\operatorname{IgG}$ (1:2000 dilution). Immune complexes were revealed by incubating with orthophenylene diamine (Sigma) and $0.15 \% \mathrm{H}_{2} \mathrm{O}_{2}$ for $30 \mathrm{~min}$. The reaction was stopped by the addition of $1 \mathrm{M} \mathrm{H}_{2} \mathrm{SO}_{4}$ and the absorbance was measured at $450 \mathrm{~nm}$ using an ELISA reader (Bio-TekEL $\times 800$, USA). All samples were run in triplicates.

Lymphoproliferation assay: Two weeks after the final inoculation, spleen cells were harvested from the seven immunized mice of each group under aseptic conditions and the erythrocytes were lysed by using $\mathrm{RBC}$ lysis solution (Sigma). Splenocytes were re-suspended in DMEM medium supplemented with $10 \% \mathrm{FCS}$, cells were plated in 96-well costar plates at a density of $5 \times 10^{5}$ cells per well and cultured with STAg $\left(\mu \mathrm{g} \mathrm{mL}^{-1}\right)$ or concanavalin A (ConA; $5 \mu \mathrm{g} \mathrm{mL}{ }^{-1}$; Sigma; positive control) or medium alone (negative control) at $37^{\circ} \mathrm{C}$ with $5 \% \mathrm{CO}_{2}$. The proliferative activity was measured using a 3-(4, 5-dimethylthylthiazol-2-yl)-2, 5-diphenyltetrazolium bromide (MTT, $5 \mathrm{mg} \mathrm{mL}^{-1}$, Sigma) dye assay, the Stimulation Index (SI) was calculated as the ratio of the average $\mathrm{OD}_{570}$ value of wells containing antigen stimulated cells to the average $\mathrm{OD}_{570}$ value of wells containing only cells with medium. All assays were performed in triplicate.
Cytokine assays: Cells were stimulated by incubation with STAg, ConA or with the medium alone. Cell-free supernatants were harvested and assayed for interleukin-2 (IL-2) and interleukin-4 (IL-4) activities at $24 \mathrm{~h}$ for interleukin-10 (IL-10) activity at $72 \mathrm{~h}$ and for gamma-interferon (IFN- $\gamma$ ) activity at $96 \mathrm{~h}$. The concentrations of IL-2, IL-4, IL-10 and IFN- $\gamma$ were evaluated by using commercial ELISA Kits according to the manufacturer's instructions (DuoSet, Genzyme). All assays were performed in triplicate. Cytokine concentrations were determined by making reference to the standard curves constructed with known amounts of mouse recombinant IFN- $\gamma, \mathrm{IL}-2, \mathrm{IL}-4$ or IL-10.

Challenge infection: Kunming mice of all groups were challenged intraperitoneally with $1 \times 10^{3}$ tachyzoites of virulent $T$. gondii ( $\mathrm{RH}$ strain) 2 weeks after the last immunization and the mice were observed and the time of the death was recorded.

Statistical analysis: All data were compared using the Statistical Package for the Social Sciences SPSS 13.0 Data Editor (SPSS Inc., Chicago, Illinosis, USA). $\mathrm{p}<0.05$ were considered to be statistically significant.

\section{RESULTS AND DISCUSSION}

Sequence variation in the MIC4 gene sequences of $T$. gondii isolates: The MIC4 fragments obtained from all examined isolates were approximately $1740 \mathrm{bp}$ in length (Fig. 1). Sequencing of purified amplicons obtained a 1743 bp sequence for all examined isolates. Sequence comparison revealed $12(0.69 \%)$ variable nucleotide positions among the examined isolates. The $\mathrm{A}+\mathrm{T}$ and $\mathrm{C}+\mathrm{G}$ contents of the sequences were 49.05-49.28 and 50.72-50.95\%, respectively. For the MП4 gene, intra-specific nucleotide variation was related mainly to

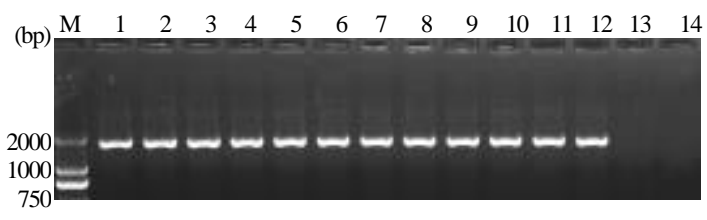

Fig. 1: Analysis of the MIC4 PCR products of Toxoplasma gondii isolates by agarose gel electrophoresis. Lanes 1 and 2 represent $\mathrm{RH}$ strain and QHO strain, respectively. Lanes 3-10 represent isolates $\mathrm{TgCtGZ1} \sim \mathrm{TgCtGZ8}$, respectively. Lanes 11 and 12 represent isolate TgPgPYS and strain $\mathrm{TgCgCa1}$, respectively. Lanes 13 and 14 represent host (mouse) and negative control, respectively. $\mathrm{M}$ represents a DNA size marker 
Table 1: Geographical origins, genotypes, hosts of Toxoplasma gondii isolates and the GenBank accession numbers of their MIC 4 genes

\begin{tabular}{lllllc}
\hline Sample codes & Host & Geographical origin & Genotype & Reference & Accession number for MIC 4 \\
\hline RH & Human & USA & I & Zhou et al., 2009 & FJ785459 \\
QHO & Sheep & Qinghai Province in China & II & Zhou et al., 2009 & FJ785460 \\
TgCtGZ1 & Cat & Guangzhou city in China & II & Zhou et al., 2009 & FJ785462 \\
TgCtGZ2 & Cat & Guangzhou city in China & II & Zhou et al., 2009 & FJ785463 \\
TgCtGZ3 & Cat & Guangzhou city in China & II & Zhou et al., 2009 & FJ785464 \\
TgCtGZ4 & Cat & Guangzhou city in China & II & Zhou et al., 2009 & FJ785465 \\
TgCtGZ5 & Cat & Guangzhou city in China & II & Zhou et al., 2009 & FJ785466 \\
TgCtGZ6 & Cat & Guangzhou city in China & II & Zhou et al., 2009 & FJ785467 \\
TgCtGZ7 & Cat & Guangzhou city in China & II & Zhou et al., 2009 & FJ785468 \\
TgCtGZ8 & Cat & Guangzhou city in China & II & Zhou et al., 2009 & FJ785469 \\
TgPgPYS & Pig & Guangzhou city in China & II & Zhou et al., 2009 & FJ785470 \\
TgCgCal & Cougar & Canada & I/IIIII & Zhou et al., 2009 & FJ785461
\end{tabular}

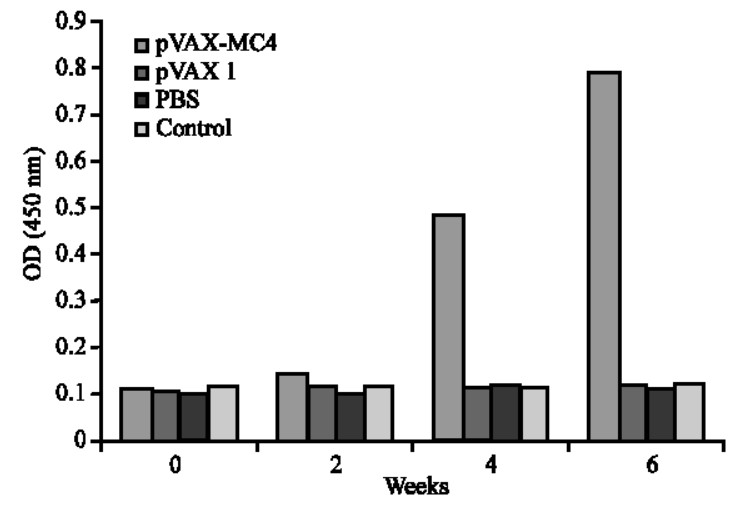

Fig. 2: Determination of specific anti-MIC4 antibody titers in the sera of Kunming mice immunized with $100 \mu \mathrm{g}$ of pVAX I or PBS alone or combined with $100 \mu \mathrm{g}$ of MIC4 on weeks 0,2 and 4. Sera were collected on 1 day prior to each immunization and tested by ELISA using STAg. The titer is given as the reciprocal of the highest dilution with an $O D_{450}$ that was 2.5 -fold greater than the OD of untreated mouse sera at the same dilution

changes at the first and second codon positions while fewer changes were detected at the third codon position (Table 1).

Identification of the expressed product by IFA: In vitro expression of pVAX-MIC4 was evaluated by IFA at $48 \mathrm{~h}$ post-transfection. The green fluorescence was observed in Marc-145 cells transfected with pVAX-MIC4.

Humoral immune responses: All sera were tested by ELISA to detect the significant $T$. gondii specific IgG production in vaccinated mice (Fig. 2). A strong antibody response was found in mice immunized with pVAX-MIC4, which were significantly higher than those of the control groups ( $\mathrm{p}<0.05)$, versus other groups).

Cytokine production: The levels of IFN- $\gamma, \mathrm{IL}-2, \mathrm{IL}-4$ and IL-10 produced in splenocytes from immunized mice are shown in Table 2. Significant high levels of IFN- $\gamma, \mathbb{L}-2$ and IL-12 were observed in spleen cell cultures from mice immunized with pVAX-MПC4 compared with mice immunized with PBS and empty plasmid. Besides, levels of IL-4 in supernatants of spleen cells from mice immunized with pVAX-MIC4 were lower than other three groups.

Protection of mice against challenge with virulent strain following DNA vaccination: To test whether immunization of pVAX-MIC4 induced effective protection against $T$. gondii infection, the immunized mice were intraperitoneally challenged with tachyzoites of the virulent $\mathrm{RH}$ strain at 2 weeks after last immunization (Fig. 3). The survival rate of the mice immunized with pVAX-MIC4 was higher than other groups $(p<0.05)$. However, immunization of Kunming mice with pVAXMIC4 only increased the survival time, which did not induce complete protection against challenge with the $\mathrm{RH}$ strain of $T$. gondii.

Previous studies have shown that MПC4 is a component of the micronemes in $T$. gondii and the deduced amino acid sequence of $\mathrm{MLC} 4$ predicts a protein that contains six conserved apple domains (Brecht et al., 2001). Microneme proteins are mainly used for host cell recognition, binding and motility. Besides, MC4 binds efficiently to host cells and the adhesive motif maps in the most C-terminal apple domain.

The present study examined nucleotide variation in MIC 4 sequences among different $T$. gondii isolates and found that the sequence variation was very low, demonstrating that $\mathrm{M \amalg C} 4$ can be considered as the candidate of DNA vaccines against $T$. gondii infection.

As an attractive alternative to conventional vaccines, DNA vaccines show promise in inducing protection against infectious diseases. Lourenco et al. (2006) showed that the vaccination of $\mathrm{C} 57 \mathrm{BL} / 6$ mice with T. gondii $\mathrm{MICl}$ and $\mathrm{MLC} 4$ induced protective immunity against infection by $T$. gondii. In the present study, the researchers used a highly-biosafety plasmid pVAX I as the vector to express MIC4, this vector contains appropriate genetic elements (e.g., promoter) required for 
Table 2: Cytokine production by splenocytes of immunized kunming mice after stimulation by STAg Cytokine production $\left(\mathrm{pg} \mathrm{mL}^{-1}\right)$

\begin{tabular}{|c|c|c|c|c|c|}
\hline Groups & IFN-y & L-2 & $\mathbb{L}-4$ & IL-10 & Proliferation SI \\
\hline pVAX-MIC4 & $657 \pm 32.74^{\mathrm{a}}$ & $614.33 \pm 30.92^{\mathrm{a}}$ & $281.33 \pm 14.29^{a}$ & $608.33 \pm 17.01^{\mathrm{a}}$ & $1.61 \pm 0.05^{\mathrm{a}}$ \\
\hline pVAXI & $52.33 \pm 11.02^{b}$ & $48.67 \pm 20.55^{b}$ & $52 \pm 15.52^{b}$ & $53.67 \pm 8.50^{b}$ & $0.19 \pm 0.03^{b}$ \\
\hline PBS & $47.33 \pm 5.13^{b}$ & $41.00 \pm 7.55^{b}$ & $50.67 \pm 4.73^{b}$ & $50.67 \pm 6.81^{b}$ & $0.20 \pm 0.06^{b}$ \\
\hline Control & $49.33 \pm 10.50^{b}$ & $45.33 \pm 8.02^{b}$ & $47.67 \pm 13.58^{b}$ & $50.00 \pm 14.18^{b}$ & $0.19 \pm 0.08^{b}$ \\
\hline
\end{tabular}

SI stands for stimulation index; Splenocytes from mice were harvested 2 weeks after the last immunization; Values for IFN- $\gamma$ are for $96 \mathrm{~h}$, values for IL-2 and IL-4 are for $24 \mathrm{~h}$, values for $\mathbb{L}-10$ are for $72 \mathrm{~h}$; The same letter means no difference $(\mathrm{p}>0.05)$ whereas different letters mean signific ant difference ( $<<0.05$ )

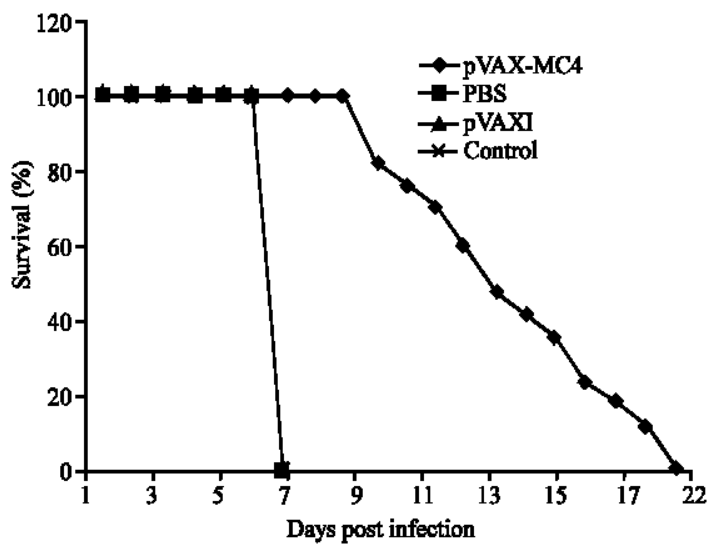

Fig. 3: Survival curves of immunized Kunming mice after lethal challenge with $1 \times 10^{3}$ tachyzoites of T. gondii $\mathrm{RH}$ strain 2 weeks after the last immunization

in vivo expression of the antigen gene of interest in the target organism and meets U.S. Food and Drug Administration (FDA) guidelines for design of DNA vaccines.

The researchers observed significant production of serum IgG antibodies (Fig. 2), a strong increase of proliferative response of splenocytes $(\mathrm{p}<0.05)$ and significant levels of IFN- $\gamma$, IL-2 and IL-10 $(\mathrm{p}<0.05)$ but low production of IL-4 compared with mice immunized with PBS or pVAX I alone (Table 2).

In this study, it is evaluated the protection induced by PVAX-MIC4 by intraperitoneally infecting the vaccinated mice with tachyzoites of the virulent $\mathrm{RH}$ strain at 2 weeks after the final immunization. An effective and highly significant degree of protection was obtained in mice immunized with DNA vaccine compared to the other groups. Mice immunized with pVAX I alone or PBS died on the fifth day. However, pVAX-MIC4 vaccination resulted in longer survival time (Fig. 3).

\section{CONCLUSION}

The present study indicated that the recombinant pVAX-MIC4 was able to elicit a significant humoral and cellular immune response, as well as increased survival time of immunized mice. These results demonstrate that that $T$. gondii MIC4 is a potential vaccine candidate against toxoplasmosis.

\section{ACKNOWLEDGEMENTS}

This study is supported in part by grants from the National Special Research Programs for Non-Profit Trades (Agriculture) (Grant No. 200803017), the Program for Changjiang Scholars and Innovative Research Team in University (Grant No. IRT0723), the Natural Science Foundation of Guangdong Province (Grant No. 9451064201003715), the State Key Laboratory of Veterinary Etiological Biology, Lanzhou Veterinary Research Institute, Chinese Academy of Agricultural Sciences (Grant No. SKLVEB2009KFKT014), the Key Research Programs in Natural Sciences for Institutions of Higher Education in Guangdong Province (Grant No. 06Z004) and the President's Funds of South China Agricultural University (Grant No. 2009K034).

\section{REFERENCES}

Angus, C.W., D. Klivington-Evans, J.P. Dubey and J.A. Kovacs, 2000. Immunization with a DNA plasmid encoding the SAG1 (P30) protein of Toxoplasma gondii is immunogenic and protective in rodents. J. Infect. Dis., 181: 317-324.

Brecht, S., V.B. Carruthers, D.J. Ferguson, O.K. Giddings and G. Wang et al., 2001. The toxoplasma micronemal protein $\mathrm{MIC4}$ is an adhesin composed of six conserved apple domains. J. Biol. Chem., 276: 4119-4127.

Buxton, D., 1998. Protozoan infections (Toxoplasma gondii, Neospora caninum and Sarcocystis sp.) in sheep and goats: Recent advances. Vet. Res., 29: 289-310.

Carruthers, V.B., O.K. Giddings and L.D. Sibley, 1999. Secretion of micronemal proteins is associated with Toxoplasma invasion of host cells. Cell. Microbiol., 1: 225-235. 
Chen, G., H. Guo, F. Lu and H. Zheng, 2001. Construction of a recombinant plasmid harbouring the rhoptry protein 1 gene of Toxoplasma gondii and preliminary observation on DNA immunity. Chin. Med. J., 114: 837-840.

Desolme, B., M.N. Mévélec, D. Buzoni-Gatel and D. Bout, 2000. Induction of protective immunity against toxoplasmosis in mice by DNA immunization with a plasmid encoding Toxoplasma gondii GRA4 gene. Vaccine, 18: 2512-2521.

Leyva, R., P. Hérion and R. Saavedra, 2001. Genetic immunization with plasmide DNA coding for the ROP2 protein of Toxoplasma gondii. Parasitol. Res., 87: $70-79$.

Lourenço, E.V., E.S. Bernardes, N.M. Silva, J.R. Mineo, A. Panunto-Castelo and M.C. Roque-Barreira, 2006. Immunization with $\mathrm{M} \mathrm{Cl}$ and $\mathrm{MIC4}$ induces protective immunity against Toxoplasma gondii. Microb. Infect., 8: 1244-1251.

Montoya, J.G. and O. Liesenfeld, 2004. Toxoplasmosis. Lancet, 363: 1965-1976.

Nielsen, H.V., S.L. Lauemøller, L. Christiansen, S. Buus, A. Fomsgaard and E. Petersen, 1999. Complete protection against lethal Toxoplasma gondii infection in mice immunized with a plasmid encoding the SAG1 gene. Infect. Immun., 67: 6358-6363.

Peng, G.H., Z.G. Yuan, D.H. Zhou, X.H. He and M.M. Liu et al., 2009. Toxoplasma gondii microneme protein 6 (MIC6) is a potential vaccine candidate against toxoplasmosis inmice. Vaccine, 27: 6570-6574.

Scorza, T., S. D'Souza, M. Laloup, J. Dewit and J. De Braekeleer et al., 2003. A GRA1 DNA vaccine primes cytolytic CD8+ $\mathrm{T}$ cells to control acute Toxoplasma gondii infection. Infect. Immun., 71: 309-316.

Strimmer, K. and A. von Haeseler, 1996. Quartet puzzling: A quartet maximum likelihood method for reconstructing tree topologies. Mol. Biol. Evol., 13: 964-969.

Thompson, J.D., T.J. Gibson, F. Plewniak, F. Jeanmougin and V. Higgins, 1997. The clustal X windows interface: Flexible strategies for multiple sequence alignment aided by quality analysis tools. Nucl. Acids Res., 25: 4876-4882.
Tomley, F.M. and D.S. Soldati, 2001. Mix and match modules: Structure and function of microneme proteins in apicomplexan parasites. Trends Parasitol., $17: 81-88$.

Vercammen, M., T. Scorza, K. Huygen, J. De Braekeleer and R. Diet et al., 2000. DNA vaccination with genes encoding Toxoplasma gondii antigens GRA1, GRA7 and ROP2 induces partially protective immunity against lethal challenge in mice. Infect. Immun., 68: $38-45$.

Wang, H., S. He, Y. Yao, H. Cong, H. Zhao, T. Li and X.Q. Zhu, 2009. Toxoplasma gondii: Protective effect of an intranasal SAG1 and MIC4 DNA vaccine in mice. Exp. Parasitol., 122: 226-232.

Weiss, L.M. and J.P. Dubey, 2009. Toxoplasmosis: A history of clinical observations. Int. J. Parasitol., 39: 895-901.

Xiang, W., Z. Qiong, L. Li-peng, T. Kui, G. Jian-wu and S. Heng-ping, 2009. The location of invasionrelated protein MIC3 of Toxoplasma gondii and protective effect of its DNA vaccine in mice. Vet. Parasitol., 166: 1-7.

Zhang, J., S. He, H. Jiang, T. Yang and H. Cong et al., 2007. Evaluation of the immune response induced by multiantigenic DNA vaccine encoding SAG1 and ROP2 of Toxoplasma gondii and the adjuvant properties of murine interleukin-12 plasmid in BALB/c mice. Parasitol. Res., 101: 331-338.

Zhou, P., H. Zhang, R.Q. Lin, D.L. Zhang, H.Q. Song, C. Su and X.Q. Zhu, 2009. Genetic characterization of Toxoplasma gondii isolates from China. Parasitol. Int., 58: 193-195.

Zhu, X.Q., S. D'Amelio, H.W. Palm, L. Paggi, M. GeorgeNascimento and R.B. Gasser, 2002. SSCP-based identification of members within the Pseudoterranova decipiens complex (Nematoda: Ascaridoidea: Anisakidae) using genetic markers in the internal transcribed spacers of ribosomal DNA. Parasitology, 124: 615-623. 